\section{Preliminary insight into the periostin leverage during periodontal tissue healing}

Padial-Molina M, Volk SL, Rios HF. Preliminary insight into the periostin leverage during periodontal tissue healing. J Clin Periodontol 2015; 42: 764-772. doi: $10.1111 /$ jcpe. 12432

\begin{abstract}
Background: Tissue repair and regeneration is assisted by the efficient coordination of cell and extracellular matrix interactions mediated by matricellular molecules such as periostin. Given its high expression around the teeth, the periodontal organ represents an ideal system to capture the protein dynamics during wound healing.

Methods: An observational prospective case-control study was designed to characterize periostin changes over time after periodontal surgery in tissue, oral fluids and serum by histological, protein and mRNA analyses.

Results: Histological analysis showed lower periostin with a diffuse local distribution pattern in disease patients. Levels of periostin in gingival crevicular fluid (GCF) increased over time for both groups, more noticeably in the periodontitis subjects. A transient and subtle change in circulating periostin levels was also noticed. The mRNA periostin levels contrasted with the protein levels and may indicate the underlying post-transcriptional regulatory process during chronic inflammation. Levels of known periodontal disease biomarkers such as IL- $\beta$, IL1- $\alpha$, TNF- $\alpha$, MIP- $1 \alpha$ and CRP served as tissue stability markers and complemented the clinical parameters recorded.

Conclusion: The transient local increase in GCF periostin after eliminating the local etiology in periodontally affected sites suggests its importance in the maturation and stability of the connective tissue. The decreasing levels observed as the tissue healed highlight its spatial/temporal significance.
\end{abstract}

\author{
Miguel Padial-Molina ${ }^{1,2}$, Sarah L. \\ Volk ${ }^{1}$ and Hector F. Rios ${ }^{1}$ \\ ${ }^{1}$ Department of Periodontics and Oral \\ Medicine, School of Dentistry, University of \\ Michigan, Ann Arbor, MI, USA; ${ }^{2}$ Department \\ of Oral Surgery and Implant Dentistry, School \\ of Dentistry, University of Granada, Granada, \\ Spain
}

Trial Registration: ClinicalTrial.gov number, NCT01180920.

Key words: periodontal disease; periodontal ligament; periodontium; periostin

Accepted for publication 8 July 2015
Periostin is a multifaceted protein implicated in numerous tissue repair and regenerative processes. Its role as a matricellular modula- tor has triggered noteworthy efforts to establish its value as a biomarker for a number of diseases (Sasaki et al. 2003, Ben et al. 2009,
Contie et al. 2010, 2011, Fujimoto et al. 2011, Okamoto et al. 2011, Nuzzo et al. 2014, Tian et al. 2014).

\footnotetext{
Conflict of interest and source of funding statement The authors declared that no conflict of interest exists. This study was funded by the NIH/NIDCR DE019872, Michigan Center for Oral Health Research (MCOHR, University of Michigan) (All), the Talentia Scholarship Program (Regional Ministry for Innovation, Science and Enterprise, Junta de Andalucía, Spain) (MPM) and the Andalucía Talent Hub Program (Andalusian Knowledge Agency, co-funded by the European Union's Seventh Framework Program, Marie Skłodowska-Curie actions [COFUND - Grant Agreement $n^{\circ} 291780$ ] and the Regional Ministry of Economy, Innovation, Science and Employment, Junta de Andalucía) (MPM). The funders had no role in study design, data collection and analysis, decision to publish or preparation of the manuscript.
} 
A few variant forms of periostin have been reported in the literature but still little is known about their specific functions (Takeshita et al. 1993, Horiuchi et al. 1999, Litvin et al. 2004, Kim et al. 2008, Bai et al. 2010). The larger, full-length 90 and $87-\mathrm{kDa}$ known isoforms are normally found to be secreted by neuroectodermal- derived fibroblasts (Horiuchi et al. 1999, Snider et al. 2008). Once it is secreted, periostin has an affinity to bind to molecules such as type I collagen, tenascin-C and BMP-1 (Kii et al. 2010, Maruhashi et al. 2010). This affinity assists in the maturation of the extracellular matrix and favours increase in tissue strength (Kii et al. 2006, Norris et al. 2007). In addition, periostin has been found to possess potent mitogenic properties via integrin receptor interactions. Its association with the $\alpha_{v} \beta_{3}$ integrin receptor has been found to support AKT/PKB signalling, increasing fibroblast and epithelial/endothelial migration as well as activating the mTOR signalling pathway leading to proliferation (Rosselli-Murai et al. 2013, Padial-Molina et al. 2014). Similar to other highly homologous proteins such as $\beta$ igH3, periostin expression is regulated by TGF- $\beta$ in response to mechanical stimulation (Rios et al. 2008). Collectively, the body of evidence regarding periostin has helped us to understand the key, pivotal role of this molecule as an orchestrator of the tissue response to environmental demands.

The study and characterization of periostin null mouse models became a turning point for this field of research. The periodontal phenotype clearly illustrated the importance of periostin in tissue stability and its response to injury. Although no human mutation resulting in complete depletion of periostin is known nor have physiological levels of periostin been reported for patients, a normal threshold that modulates the biomechanical competency of the periodontal extracellular matrix (ECM) can be assumed. Interestingly, the primary aetiological factors for the most common human inflammatory condition that affects the adult dentition has a suppressive effect on periostin. Both bacterial byproducts and inflammatory cytokines exert a negative effect on periostin expression by periodontal ligament (PDL) fibroblasts and as a consequence, defects in the ECM occur and compromise the structural and functional integrity of the tooth-supporting apparatus (Padial-Molina et al. 2012b, 2013). Therefore, an increase in periostin levels after surgical treatment or by active delivery can be hypothesized to improve periodontal wound healing and regeneration due to its properties in stabilizing the ECM and promoting periodontal tissue cell activity. Subsequently, the human periodontal disease condition serves as an ideal model to learn how neuroectodermalderived cells such as the periodontal fibroblasts cope with the trauma associated with surgery during the initial phases of wound healing and support a periostin expression profile that moves the healing process forward.

The overarching goal of this study was to capture the periostin expression profile that favours wound stability and maturation. The periodontal disease condition serves as the chronic burden that challenges the host and prevents proper matricellular function and therefore favouring a long-lasting infection. Our study is designed to characterize the expression of periostin in health and disease and to analyze its chronological dynamics during wound healing resolution.

\section{Methods}

\section{Trial design}

An observational prospective casecontrol study was designed and approved by the University of Michigan Institutional Review Board. The trial is registered at ClinicalTrials.gov, number NCT01180920

\section{Participants (Inclusion and exclusion)}

Subjects from the Michigan Center for Oral Health Research (MCOHR) were evaluated after obtaining the appropriate informed consent. Adults aged 21 years and above of both genders were eligible for the study. For disease subjects (case group), a diagnosis of generalized chronic or aggressive periodontitis, presence of at least four periodontal sites with probing depth (PD) $\geq 6 \mathrm{~mm}$, evidence of clinical attachment loss (CAL), $>10 \%$ of sites with bleeding on probing (BOP) and need of an open flap debridement were established as the inclusion criteria. Inclusion criteria for healthy individuals (control group) included PD $<4 \mathrm{~mm}$, no evidence of attachment loss, $<10 \%$ of sites with BOP and need of a gingivectomy or crown lengthening procedure. Candidates were excluded if they presented history of alcoholism or drug abuse; medical conditions that may affect the outcome of periodontal surgery such as autoimmune diseases, diabetes, immunocompromised subjects or systemic infections; chronic medications known to affect the periodontal status such as calcium antagonists, anticonvulsives, immunosuppressives, anti-inflammatory medications or contraceptives; antibiotic therapy within 3 months of the baseline visit; antibiotic therapy needed for infective endocarditis prophylaxis; current use or those who quit smoking less than 6 months ago with a pack-year history of more than or equal to 10 ; current dental conditions, including orthodontic treatment (presence of orthodontic devices), insufficient keratinized gingival (equal or less than $2 \mathrm{~mm}$ ), untreated carious lesions or defective restorations which could exacerbate.

A total of 185 patients showed interest in participating in the study and were assessed for eligibility. Eighty two did not meet the inclusion criteria, 13 declined to participate and 68 were excluded for other reasons, including scheduling concerns, other concomitant treatment needs that would be better treated in the same procedure and patients already participating in any other studies. About 22 patients were enrolled in this study, 11 in need of a gingival flap and tooth root instrumentation (chronic periodontal inflammation/infection group) and 11 in need of a gingival re-contouring procedure (non-inflamed/Periodontally stable group). None of them were lost to follow-up, discontinued the treatment for any reason or suffered any adverse event. Figure 1a represents the CONSORT flow diagram of patients in the study.

Patient demographics and main variables distribution were homoge- 


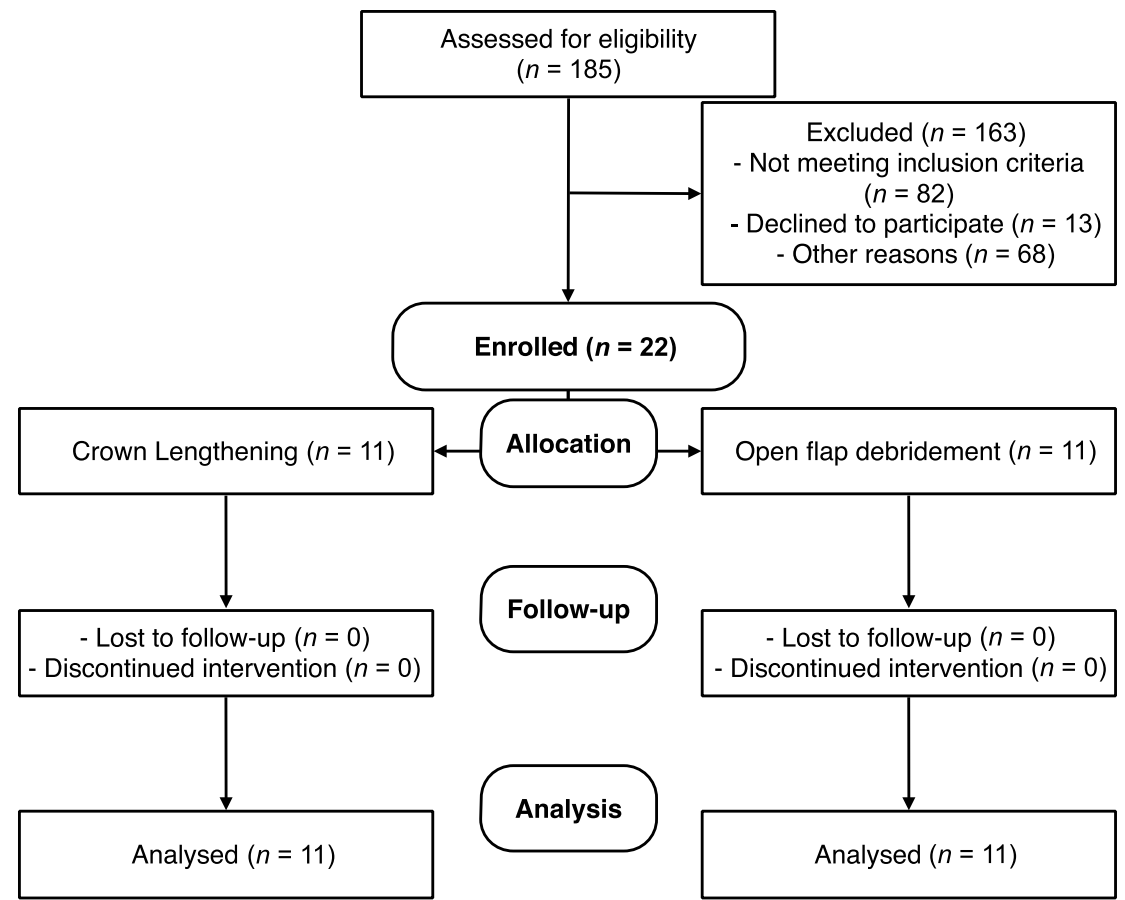

(a)

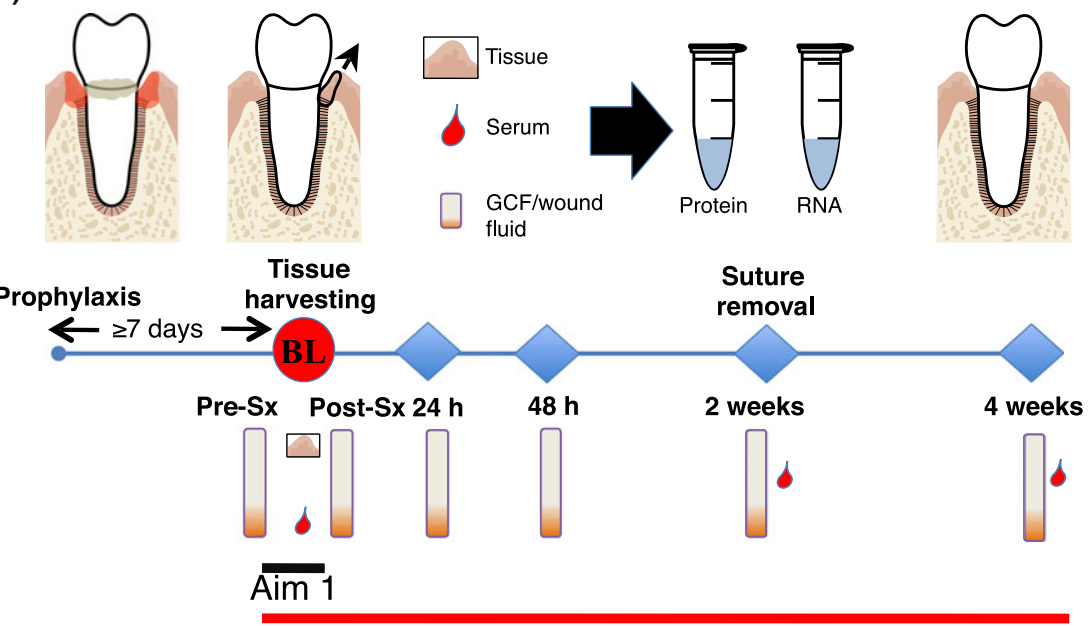

Aim 2

(b) Prophylaxis = Supragingival dental cleaning; $\mathrm{BL}=$ Baseline; $\mathrm{Sx}=$ Surgical procedure

Fig. 1. Study design. (a) CONSORT diagram of study participants. (b) Diagram of study procedures.

neous between the two groups and representative of the local population in terms of ethnic group (selfreported) (Table 1). No significant differences were found among groups for any of these variables.

\section{Study timeline and sample collection}

At baseline, tissue biopsies were collected from the selected sites during the necessary periodontal surgery. Additionally, GCF/wound fluid (immediately before and after the procedure), whole saliva (WS) and serum were collected. Patients were prescribed an over-the-counter analgesic and an antibiotic when needed. Oral and written post-surgical recommendations were given. After the surgery, GCF/wound fluid and WS samples were collected at $24 \mathrm{~h}, 48 \mathrm{~h}, 2 \mathrm{wk}$ and $4 \mathrm{wk}$. Additional serum samples were collected at $2 \mathrm{wk}$ and $4 \mathrm{wk}$. See Fig. $1 \mathrm{~b}$ for study timeline.
Refer to Appendix S1 for complete sample collection and analysis methodology.

\section{Outcomes}

The samples were processed appropriately to analyse each one of the different variables. Different portions of tissue were analysed by immunohistochemistry (for periostin protein localization), ELISA (for protein levels of periostin) or qRTPCR (for levels of periostin mRNA). Protein levels of periostin were also analysed by ELISA in GCF/wound fluid, WS and serum. In addition, $\mathrm{GCF}$ /wound fluid was also used for the analysis of periodontal diseaserelated cytokines (CRP, IL- $1 \alpha$, IL-1ß, IL-6, IL-8, IL-10, MIP-1 $\alpha$, Osteopontin, Osteoactivin, MMP-9, MMP-13 and TNF- $\alpha$ ) by using an array-based multiplex ELISA system. Finally, clinical measurements were used to evaluate changes over time in PD, CAL, oral hygiene, discomfort, soft tissue index and wound healing index.

\section{Statistical methods (Power analysis)}

An inter-rater reliability analysis using the Kappa statistic was performed to determine consistency among raters for all clinical parameters. Additionally, intra-rater testretest reliability was also assessed by Pearson's correlation test. Demographic data is reported as prevalence of categorical data and differences analysed by Pearson's $\chi^{2}$ test. Unless otherwise specified, continuous data is reported as mean (SD). Comparisons within groups were made by paired $t$-test. Comparisons among groups were made by independent $t$ test. Additionally, to analyse differences within groups over time for periostin in GCF/wound fluid and serum, a repeated measures ANOVA test was designed (with GreenhouseGeisser correction if a significant Mauchly's test indicates a violation of sphericity). Level of statistical significance was established at $p<0.05$. Given that periostin has never been evaluated in oral fluids, we chose to extrapolate findings from other disease entities. We have identified the effect size as part of the initial power analysis to establish a proper sample size estimated to be $20 \mathrm{ng} / \mathrm{ml}$ with $\alpha$ 
value of 0.05 and $80 \%$ power. This determination was based on periostin levels reported in the cancer literature from healthy patients $(\sim 30 \mathrm{ng} / \mathrm{ml})$ and diseased patients $(\sim 50 \mathrm{ng} / \mathrm{ml})$.

\section{Results}

\section{Clinical results}

Examiner calibration between the examiner and the standard examiner showed a $90.68 \%$ of exact agreement pairs with a $\kappa=0.876(95 \%$ CI, 0.944 to 0.807$)(p<0.0005)$ for PD and a $63.56 \%$ of exact agreement pairs with a $\kappa=0.552(95 \%$ CI, 0.651 to 0.452$)(p<0.0005)$ for CAL. Intra-examiner reliability was $100 \%$ for both the PD and CAL.

Clinical measurements (in $\mathrm{mm}$ ) showed statistically significant differences for independent comparisons of PD [3.0 (0.9) versus 6.7 (1.9); 2.3 (0.8) versus $3.9(1.0)]$ and CAL [2.3 (0.9) versus 6.5 (2.0); 2.8 (0.6) versus 4.8 (1.3)] (healthy versus disease at baseline and 4 weeks respectively), and PD change $[0.7$ (1.0) versus 2.8 (1.3)] and CAL change [-0.5 (1.1) versus 1.7 (2.0)] (healthy versus disease) $(p<0.001$, Independent $t$-test). Within groups, PD at baseline was significantly different than PD at 4 weeks (paired $t$-test) for both healthy $(p<0.038)$ and disease groups $(p<0.0005)$, and CAL from baseline to 4 weeks in the disease group ( $p<0.015)$ (Fig. 2).

As expected, qualitative clinical variables showed a progressive improvement over time for all variables analysed (Oral hygiene, Discomfort, Soft tissue index and Wound healing index) (Fig. S1). Oral hygiene worsened from $24 \mathrm{~h}$ to $48 \mathrm{~h}$, which was expected as patients were instructed to not brush the area of the surgery during the first 5 days.

\section{Periostin in tissue}

Histological analysis of the tissues collected from the healthy periodontium during the surgical procedures (Fig. 3) showed that the periostin immunoreactivity signal highlights a robust distribution and localization of the secreted protein in the underlying specialized connective tissue right beneath the junctional epithelium. A fibrillar pattern of periostin distribution was observed. Such observation is similar to the fibrillar structure and directionality of the periodontal ligament to the alveolar bone and the tooth root surface. The hematoxylin \& eosin (H\&E) staining showed no relevant inflammatory infiltrate but the normal characteristics of a healthy epithelium, junctional epithelium and connective tissue. In contrast, the tissue samples from periodontal disease

Table 1. Demographic variables

\begin{tabular}{lccc}
\hline & Healthy $-n=11$ & Disease $-n=11$ & Pearson's $\chi^{2}$ \\
\hline Age - years & & & \\
$\quad$ Median & 39 & 51 & 0.232 \\
$\quad$ Range & $22-70$ & $29-71$ & \\
Sex $-n(\%)$ & $8(73)$ & $7(64)$ & 0.647 \\
$\quad$ Male & $3(27)$ & $4(36)$ & \\
Female & & & \\
Race or ethnic group $-n(\%)^{*}$ & $8(73)$ & $9(82)$ & 0.383 \\
$\quad$ Caucasian & $1(9)$ & $1(9)$ & \\
African American & $0(0)$ & $1(9)$ & \\
Asian & $2(18)$ & $0(0)$ & \\
Hispanic & $0(0)$ & $0(0)$ & \\
Native American & $0(0)$ & $0(0)$ & \\
Other & $7(64)$ & $7(64)$ & 1.000 \\
Smoking status $-n(\%)$ & $4(36)$ & $4(36)$ & \\
Never Smoked & & & \\
Former Smoker $(>6$ months) & & & \\
\end{tabular}

* Race or ethnic group was self-reported.

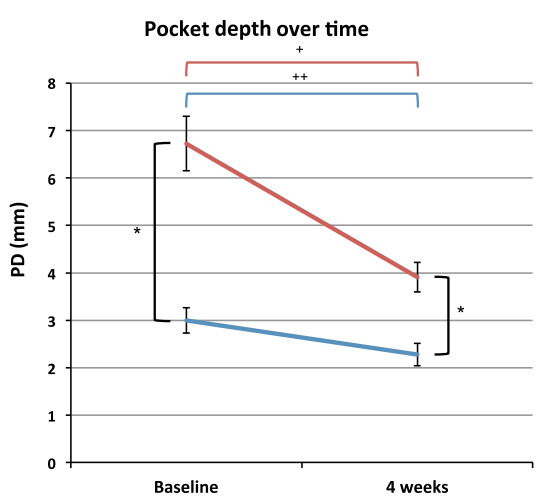

(a)

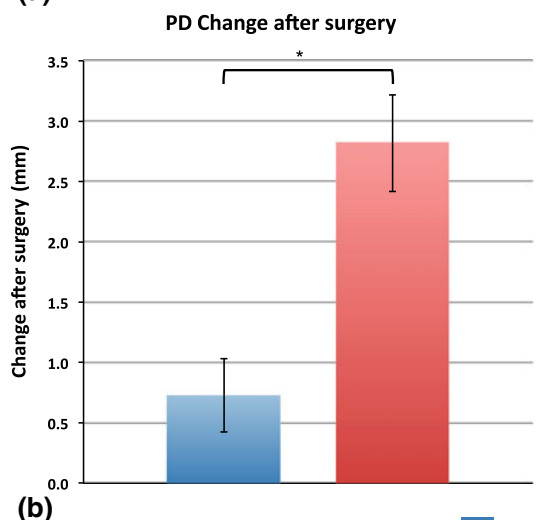
Healthy

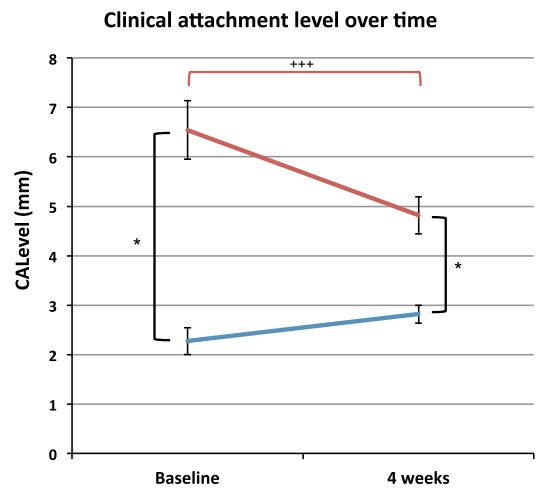

(c)

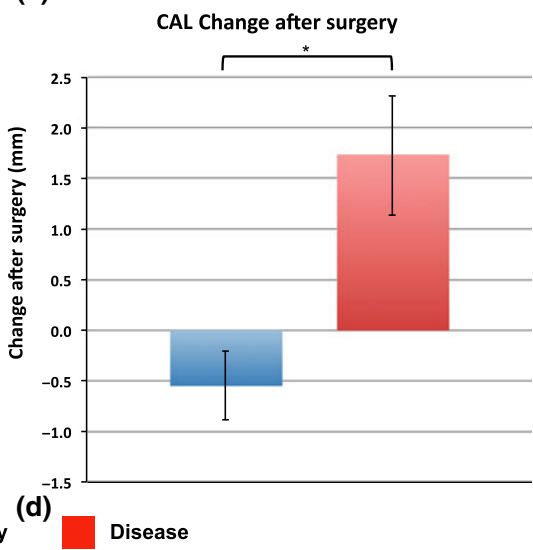

Fig. 2. Clinical measurements (mean \pm SEM) of (a) Probing depth and (b) Clinical attachment level at baseline and 4 weeks, as well as (c) Probing depth and (d) Clinical attachment level change from baseline to 4 weeks. ${ }^{*} p<0.001$ (Independent $t$-test); ${ }^{+} p<0.001 ;{ }^{++} p<0.038 ;{ }^{+++} p<0.015$ (Paired $t$-test). 


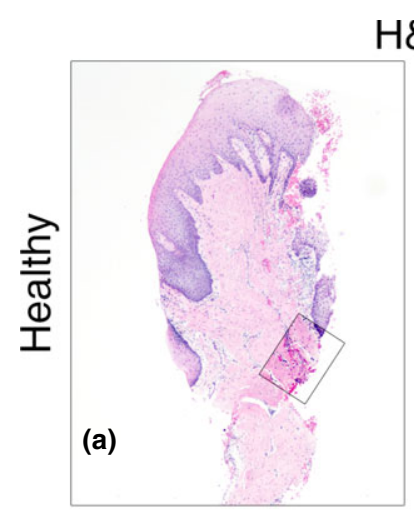

$H \& E$
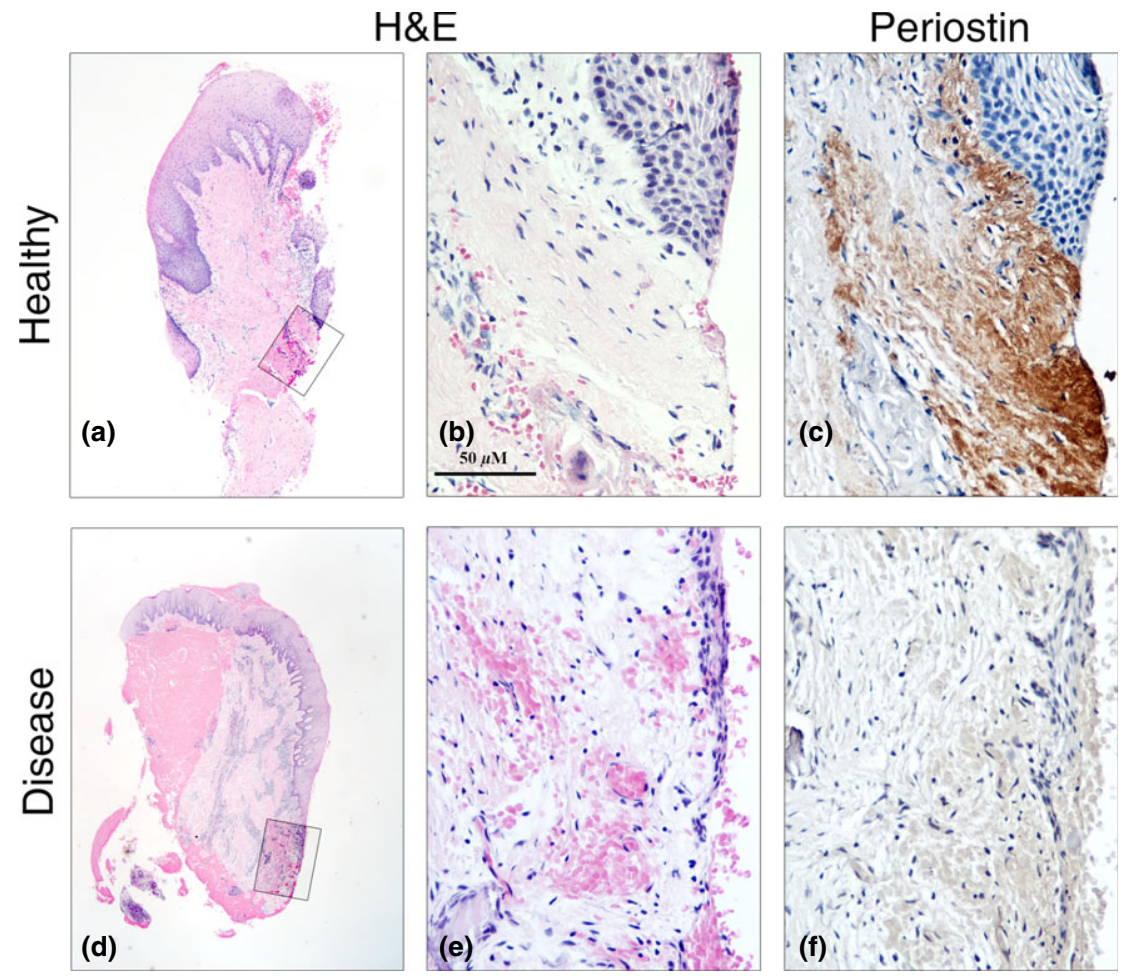

Fig. 3. Representative images of the tissue histological analysis for both healthy (a, b, c) and disease patients (d, e, f) by hematoxylin-eosin (a, b, d, e) and periostin stainings (c, d) captured at an original magnification of $2 \times(\mathrm{a}, \mathrm{d})$ and $40 \times(\mathrm{b}, \mathrm{c}, \mathrm{e}, \mathrm{f})$ in the area of interest (insert in a and $\mathrm{d}$ below the junctional epithelium).

patients showed overall lower and diffuse pattern of extracellular periostin incorporation in the area beneath the functional epithelium. As expected, a localized chronic inflammatory infiltrate front was visible in the H\&E sections and colocalized with the areas of diffuse periostin signal.

The whole tissue lysates contrasted with the detailed morphological description of periostin protein distribution feasible by the available tissue sections. As a limitation determined by the biopsy collection method, when assessing periostin protein levels in the whole tissue sample lysate (in $\mathrm{ng} / \mathrm{ml}$ ), those spatial differences were washed away and no statistical significant differences comparing the healthy [ 42.559 (23.696)] and the disease samples were observed [38.133 (19.631)] $(p=$ 0.639 , Independent $t$-test) (Fig. S2). Similarly, mRNA analysis of the whole tissue lysate showed no statistically significant differences of periostin [1.00 (0.87) versus 1.902 (2.20); $p=0.221], \quad \beta I G-H 3 \quad[1.00 \quad(0.73)$ versus $0.951(0.67) ; p=0.870]$, collagen I [1.00 (0.61) versus 2.51 (3.92); $p=0.233]$, Twist $[1.00(0.64)$ versus $1.46(0.94) ; p=0.192]$ and TGF- $\beta 1$

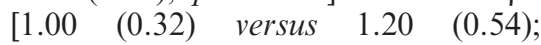
$p=0.306]$ when comparing healthy

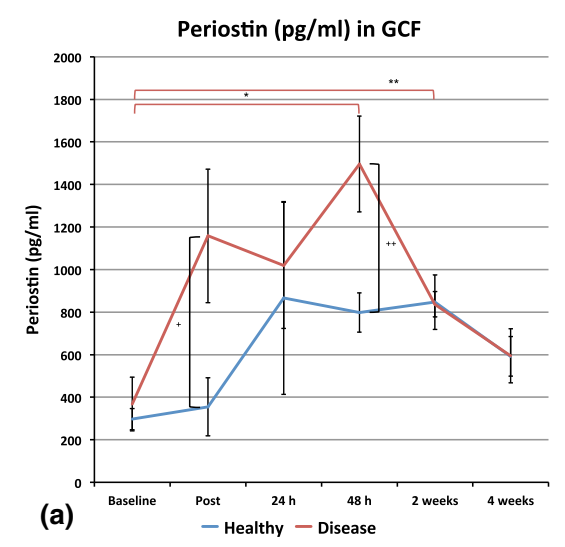
hoc)]. versus disease groups (Independent $t$-test) (Fig. S3).

\section{Periostin protein in GCF/wound fluid and} serum

Levels of periostin in $\mathrm{GCF} /$ wound fluid (in $\mathrm{pg} / \mathrm{ml}$ ) (Fig. 4a) increased over time and correlated with the healing process in both healthy and disease patients. A repeated measures ANOVA with a GreenhouseGeisser correction [Mauchly's Test of Sphericity: $\chi^{2} \quad(14)=28.947$, $p<0.013$ ] determined that mean periostin concentration in the disease group was statistically significant between time points $[F \quad(5.252$, $27.246)=2.725, p<0.007]$. Post hoc tests using the Bonferroni correction revealed that periostin levels previous to the surgery [367.85 (418.87)] were statistically significantly lower than those at $48 \mathrm{~h}[1496.14$ (747.48), $p<0.001]$ and 2 weeks $[836.80$ (199.606), $p<024]$. In healthy subjects, the repeated measures ANOVA with a Greenhouse-Geisser correction [Mauchly's Test of Sphericity: $\left.\chi^{2}(14)=70.908, p<0.0005\right]$ showed no statistically significant differences over time $[F(1.226,12.264)=1.829$, $p=0.203]$ and therefore, post hoc comparisons were not performed. Differences among groups were statistically significantly different only immediately post-surgery $[354.68$ (452.22) versus $1158.31 \quad$ (1041.72); $p<0.029]$ and at $48 \mathrm{~h} \quad[798.24$ (306.47) versus 1496.14 (747.48);

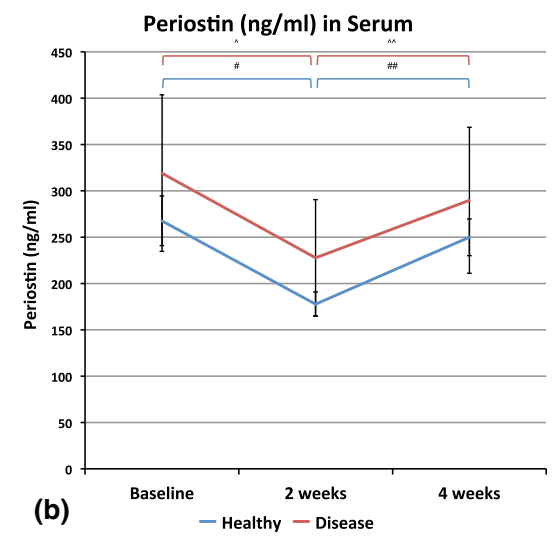

Fig. 4. Levels of periostin (mean \pm SEM) over time for healthy (blue) and diseased (red) patients expressed as mean $\pm \mathrm{SEM}$ in (a) GCF/wound fluid (pg/ml) $\left[{ }^{*} p<0.001\right.$; ${ }^{* *} p<0.024$ (Bonferroni post hoc); ${ }^{+} p<0.029 ;{ }^{++} p<0.013$ (Independent $t$-test] and (b) serum (ng/ml) $\left[{ }^{\#} p<0.001 ;{ }^{\# \#} p<0.0005 ;{ }^{\wedge} p<0.015 ;{ }^{\wedge} \hat{p} p<0.020\right.$ (Bonferroni post 
$p<0.013$ ] (healthy versus disease; Independent $t$-test).

Periostin levels in serum (Fig. 4b) showed a reduction from baseline [267.58 (88.77) versus 319.04 (280.43); $p=0.568]$ to 2 weeks [177.64 (43.26) versus 227.59 (207.96); $p=0.445]$ and went back to initial values at 4 weeks [249.79 (65.76) versus 289.69 (261.07); $p=0.628]$ (healthy versus disease; Independent $t$-test) in both groups. Repeated measures ANOVA with sphericity assumption [Mauchly's Test of Sphericity: $\chi^{2}(2)=2.061$, $p=0.357]$ determined that the mean periostin concentration in serum in the healthy group differed statistically significant between time points $[F(2$, $20)=21.122, p<0.0005]$. Post hoc tests using the Bonferroni correction revealed that periostin levels at 2 weeks were statistically significantly lower to those detected prior to the surgery $(p<0.001)$ and at 4 weeks $(p<0.0005)$. Similarly, repeated measures ANOVA with a Greenhouse-
Geisser correction [Mauchly's Test of Sphericity: $\left.\chi^{2}(2)=5.022, p=0.081\right]$ determined that mean periostin concentration in serum in the disease group differed statistically significantly between time points $[F$ $(1.401,14.009)=10.914, p<0.003]$. Post hoc tests using the Bonferroni correction revealed that periostin levels at 2 weeks were statistically significantly lower to those detected prior to the surgery $(p<0.015)$ and at 4 weeks $(p<0.020)$.

Periostin protein was undetectable in saliva in the present study.

\section{Periodontal disease-related cytokines in serum and GCF/wound fluid}

The CRP in serum (Fig. S4) and periodontal disease-related cytokines in GCF/wound fluid (Fig. 5) follows a similar pattern as in other previously published studies (Kinney et al. 2007). In this sense, it is interesting to note the differences between baseline and 4-weeks time points for CRP, IL$1 \alpha$, IL-1 $\beta$, and TNF- $\alpha$, specifically in disease patients. Those levels became similar to those obtained in healthy patients, indicating the resolution of the disease process.

\section{Discussion}

Besides the periodontium, the matricellular molecule periostin has been recognized as a pivotal participant in the functional stability of multiple systems. In general, it has been shown that the larger, full-length 90 and $87-\mathrm{kDa}$ isoforms are normally secreted by cells such as neuroectodermal-derived fibroblasts (Horiuchi et al. 1999, Snider et al. 2008). Tissue-specific isoforms are thought to be modulators of respective tissues. In the PDL, the large full-length variants have been reported to regulate FAK signalling and potentially influenced mineralization when over
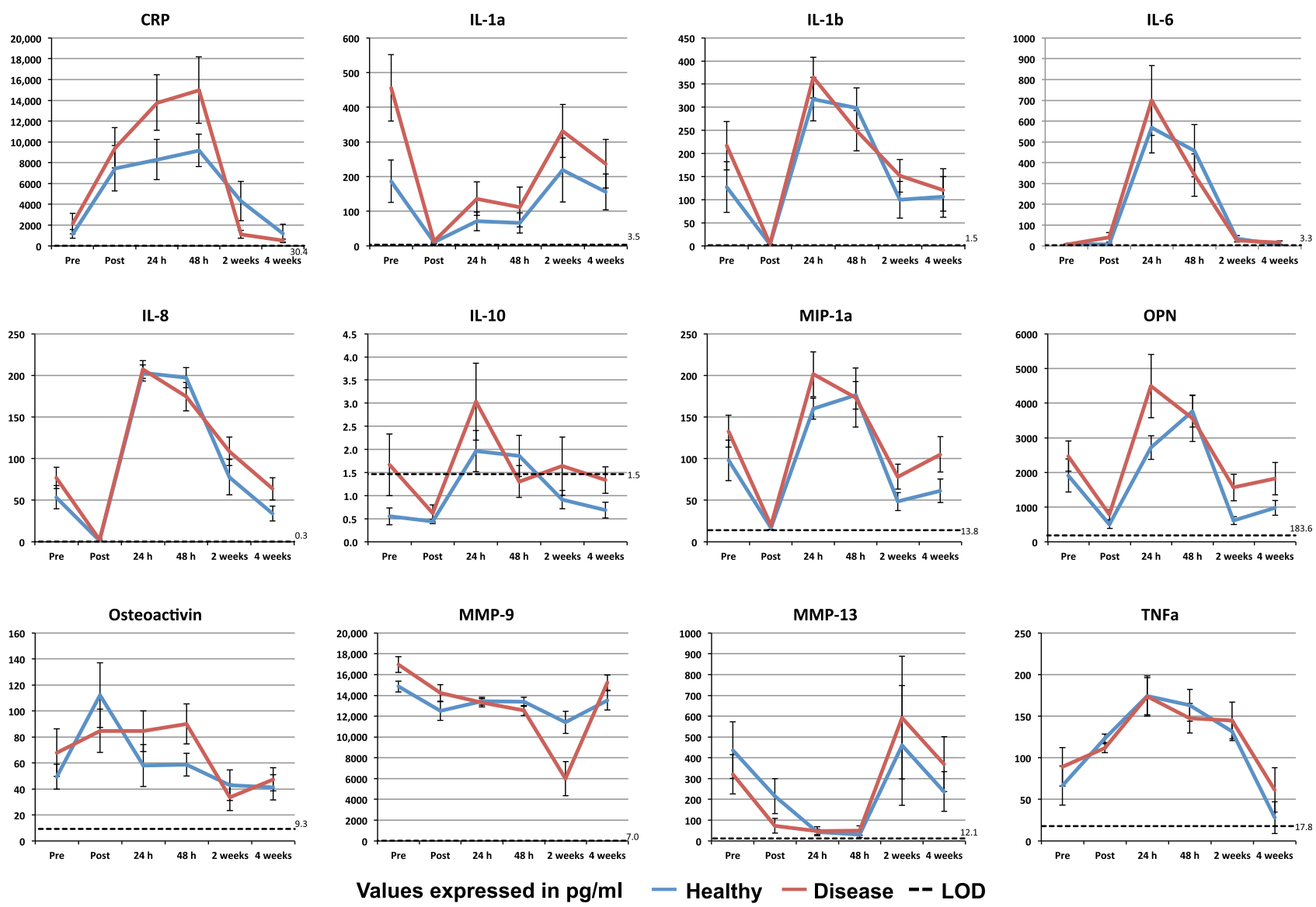

Fig. 5. Levels of periodontal disease markers over time in GCF/wound fluid in $\mathrm{pg} / \mathrm{ml}$ (mean \pm SEM) for healthy (blue) and disease (red) patients. 
expressed in vitro (Yamada et al. 2014). It is speculated that these isoforms are downstream responders along the PDL differentiation pathway. Therefore, in the context of wound healing after surgery, its expression profile should be transient (i.e. switched off after differentiation or as PDL inflammation diminishes) while if it stays expressed, normal tissue function could be compromised (Conway et al. 2014).

The timely and spatially sensitive regulation of matricellular interactions determines the adaptive, reparative and regenerative response by modulating function among important growth factors, cytokines and proteases (Hamilton 2008), therefore, allowing proper healing and function. The currently known functional features of periostin together with the findings of this study supports that this matricellular molecule does not passively follow the extracellular matrix response to trauma. Instead, it seems to critically modulate inflammatory and reparative pathways by transducing signals that affect cell survival, migration and matrix maturation along with phenotype and gene expression at active sites of tissue repair and regeneration. Similarly to what has been seen in the injured heart (Conway \& Molkentin 2008), in the periodontium, periostin is released by specialized fibroblasts to regulate cellular interactions and promote matrix organization.

Of the known proteins expressed in the PDL, periostin shows the greatest specificity (Saito et al. 2002). It is localized between the cytoplasmic processes of periodontal fibroblasts and cementoblasts and the adjacent collagen fibrils (Suzuki et al. 2004). It has been, therefore, used as a marker of successful periodontal regeneration (Park et al. 2012, 2014). When absent in KO mice, a periodontal disease-like phenotype compared to wild-type animals can be observed (Rios et al. 2008). Similarly, periodontal disease causal factors reduce the localization of periostin in the periodontal ligament, both in vitro (Padial-Molina et al. 2013) and in vivo (PadialMolina et al. 2012b). However, acute exposure to the same pro-inflammatory factors (TNF- $\alpha$ and $P$. gingivalis
LPS) seems to induce its secretion from gingival fibroblasts (Nakajima et al. 2014). The different cell populations involved in each phase of the disease may explain this apparent discrepancy. At the gingival epithelial level, periostin promotes an increased proliferation and migration rate similar to that observed in skin epithelial cells as a potential enhancer of wound closure after injury (Rosselli-Murai et al. 2013). Similarly, epithelial cells, as the first front of defence in periodontal tissues, increase periostin release to promote gingival sealing around the teeth in response to bacteria. In contrast, as the disease progresses, the inflammatory process evolves and reaches the sub-gingival connective tissue and periodontal ligament. Then, those factors promote a reduction of periostin, which additively weakens the tissue and, therefore, disease progression is accelerated. According to our results, differences in periostin localization in the tissue by immunohistological analysis were present (Fig. 3). However, analyses of the gingival fluid (Fig. 4a) or the whole tissue lysate (Figs S2 and S3) at baseline did not show such differences, possibly due to the masking effects induced by other surrounding tissues. Additionally, patients included in this study presented different types and severities of the disease. Consequently, the use of periostin as a marker of periodontal disease could not be concluded from the present study, although a recent report suggests this correlation (Balli et al. 2014); the study showed that periostin levels in gingival crevicular fluid decreased proportionally with the progression and severity of periodontal disease, and negatively correlated with the clinical parameters. Interestingly, periostin in serum was higher in diseased patients, which correlates with our findings of an inverse correlation between periostin levels in serum and GCF/ wound fluid. Further studies are still needed, specifically in aggressive cases, where some differences were observed in GCF/wound fluid.

Periodontal wound healing and homeostasis requires the migration to the area of specific cells, at a specific time, in a specific sequence (Padial-Molina et al. 2012a). Periostin is essential in these processes
(Hamilton 2008) by regulating collagen fibrillogenesis, which influences tissue mechanical stability and strength (Norris et al. 2007). Moreover, periostin activates the cell survival AKT/PKB/mTOR pathway (Rosselli-Murai et al. 2013, PadialMolina et al. 2014) and induces migration and proliferation through the interaction with $\alpha \mathrm{v} \beta 3$ and $\alpha \mathrm{v} \beta 5$ integrins ( $\mathrm{Li}$ et al. 2010, Utispan et al. 2012). Similar effects have been previously reported in other tissues, including skin (Nishiyama et al. 2011), heart (Dorn 2007, Kuhn et al. 2007, Polizzotti et al. 2012), during angiogenesis (Zhu et al. 2010) and metastasis (Wang \& Ouyang 2012). Therefore, restoration of proper levels of this molecule in the PDL environment may have key implications in the restoration of its original structure and function, specifically after periodontal surgery, with potential use as a regenerative agent. Our results show a statistically significant increase of periostin after periodontal surgery in $\mathrm{GCF} /$ wound fluid, being greater in disease patients (Fig. 4a). This increase is explained by the reduction of chronic inflammatory stimuli and bacterial challenge as a consequence of surgical procedure. Periostin increases temporarily to assist in the healing process by increasing cell migration and proliferation, and in the establishment of a stable extracellular matrix. As the wound matures, levels of periostin detectable in $\mathrm{GCF} /$ wound fluid are reduced back to baseline levels, possibly due to a higher rate of deposit of periostin in the extracellular matrix as the collagen structure matures. Similar effects have been previously observed in vitro (PadialMolina et al. 2013).

In contrast, periostin in serum is significantly reduced by 2 weeks and back to baseline levels at 4 weeks following a similar pattern in both healthy and disease patients (Fig. 4b). Serum levels of periostin are currently under study in a wide variety of systemic diseases, including asthma (Matsumoto 2014), myocardial infarction (Ling et al. 2014), atherosclerosis (Hakuno et al. 2010), atopic dermatitis (Kou et al. 2014), bone metabolism (Rousseau et al. 2014), different cancers and others. This reflects the implications of systemic periostin in a 
number of pathologies broader than the limited localized surgery performed in our study. However, the significant changes observed over time in the present study, in the absence of any other change in the patients' systemic conditions, reflects the influence of the periodontal status on the levels of periostin being released into the blood stream, which may have important implications in distant tissues and systemic pathologies.

The study of periostin thus provides a unique opportunity to learn more about healing dynamics in different conditions. Extrapolating the findings reported in this study could have significant implications in aiding our understanding of cell-matrix dynamics and homeostasis in different systems. Unravelling this periostin-mediated matricellular pathway provides an opportunity to explore and could ultimately help us to develop practical therapeutic protocols that may impact and predictably favour regeneration over repair wound healing outcomes across different human conditions.

\section{Conclusion}

The increase in periostin levels over time in GCF/wound fluid suggests a potential implication in the healing process after periodontal surgery in patients. Periostin follows a transient but pronounced increase after surgery in periodontally affected subjects that is maintained for 2 weeks but appears to peak at $48 \mathrm{~h}$. This expression profile suggests a role for this molecule on moving the granulation phase of wound healing forward where recruitment, angiogenesis and proliferative events are essential to provide a better local environment that leads the wound healing process into optimal tissue maturation and wound stabilization.

\section{Acknowledgements}

The authors thank all the staff members at the Michigan Center for Oral Health Research for their support during the clinical phases of the study.

\section{References}

Bai, Y., Nakamura, M., Zhou, G., Li, Y., Liu, Z., Ozaki, T., Mori, I. \& Kakudo, K. (2010)
Novel isoforms of periostin expressed in the human thyroid. Japanese Clinical Medicine 1 , 13-20.

Balli, U., Keles, Z. P., Avci, B., Guler, S., Cetinkaya, B. O. \& Keles, G. C. (2014) Assessment of periostin levels in serum and gingival crevicular fluid of patients with periodontal disease. Journal of Periodontal Research. doi:10.1111/ jre.12254.

Ben, Q. W., Zhao, Z., Ge, S. F., Zhou, J., Yuan, F. \& Yuan, Y. Z. (2009) Circulating levels of periostin may help identify patients with more aggressive colorectal cancer. International Journal of Oncology 34, 821-828.

Contie, S., Voorzanger-Rousselot, N., Litvin, J., Bonnet, N., Ferrari, S., Clezardin, P. \& Garnero, P. (2010) Development of a new ELISA for serum periostin: evaluation of growth-related changes and bisphosphonate treatment in mice. Calcified Tissue International 87, 341350

Contie, S., Voorzanger-Rousselot, N., Litvin, J. Clezardin, P. \& Garnero, P. (2011) Increased expression and serum levels of the stromal cellsecreted protein periostin in breast cancer bone metastases. International Journal of Cancer $\mathbf{1 2 8}$ 352-360.

Conway, S. J., Izuhara, K., Kudo, Y., Litvin, J., Markwald, R., Ouyang, G., Arron, J. R., Holweg, C. T. \& Kudo, A. (2014) The role of periostin in tissue remodelling across health and disease. Cellular and Molecular Life Sciences 71, 1279-1288.

Conway, S. J. \& Molkentin, J. D. (2008) Periostin as a heterofunctional regulator of cardiac development and disease. Current Genomics $\mathbf{9}$, 548-555.

Dorn, G. W. 2nd (2007) Periostin and myocardial repair, regeneration and recovery. New England Journal of Medicine 357, 1552-1554.

Fujimoto, K., Kawaguchi, T., Nakashima, O., Ono, J., Ohta, S., Kawaguchi, A., Tonan, T., Ohshima, K., Yano, H., Hayabuchi, N., Izuhara, K. \& Sata, M. (2011) Periostin, a matrix protein, has potential as a novel serodiagnostic marker for cholangiocarcinoma. Oncology Reports 25, 1211-1216.

Hakuno, D., Kimura, N., Yoshioka, M., Mukai, M., Kimura, T., Okada, Y., Yozu, R., Shukunami, C., Hiraki, Y., Kudo, A., Ogawa, S. \& Fukuda, K. (2010) Periostin advances atherosclerotic and rheumatic cardiac valve degeneration by inducing angiogenesis and MMP production in humans and rodents. $J$ Clin Invest 120, 2292-2306.

Hamilton, D. W. (2008) Functional role of periostin in development and wound repair: implications for connective tissue disease. $J$ Cell Commun Signal 2, 9-17.

Horiuchi, K., Amizuka, N., Takeshita, S., Takamatsu, H., Katsuura, M., Ozawa, H., Toyama, Y., Bonewald, L. F. \& Kudo, A. (1999) Identification and characterization of a novel protein, periostin, with restricted expression to periosteum and periodontal ligament, and increased expression by transforming growth factor beta. Journal of Bone and Mineral Research 14, 1239-1249.

Kii, I., Amizuka, N., Minqi, L., Kitajima, S., Saga, Y. \& Kudo, A. (2006) Periostin is an extracellular matrix protein required for eruption of incisors in mice. Biochemical and Biophysical Research Communications 342, 766772.

Kii, I., Nishiyama, T., Li, M., Matsumoto, K., Saito, M., Amizuka, N. \& Kudo, A. (2010) Incorporation of tenascin-C into the extracellular matrix by periostin underlies an extracellular meshwork architecture. Journal of Biological Chemistry 285, 2028-2039.

Kim, C. J., Isono, T., Tambe, Y., Chano, T., Okabe, H., Okada, Y. \& Inoue, H. (2008) Role of alternative splicing of periostin in human bladder carcinogenesis. International Journal of Oncology 32, 161-169.

Kinney, J. S., Ramseier, C. A. \& Giannobile, W. V. (2007) Oral fluid-based biomarkers of alveolar bone loss in periodontitis. Annals of the New York Academy of Sciences 1098, 230-251.

Kou, K., Okawa, T., Yamaguchi, Y., Ono, J., Inoue, Y., Kohno, M., Matsukura, S., Kambara, T., Ohta, S., Izuhara, K. \& Aihara, M. (2014) Periostin levels correlate with disease severity and chronicity in patients with atopic dermatitis. British Journal of Dermatology 171, 283-291.

Kuhn, B., del Monte, F., Hajjar, R. J., Chang, Y. S., Lebeche, D., Arab, S. \& Keating, M. T (2007) Periostin induces proliferation of differentiated cardiomyocytes and promotes cardiac repair. Nature Medicine 13, 962-969.

Li, G., Jin, R., Norris, R. A., Zhang, L., Yu, S., Wu, F., Markwald, R. R., Nanda, A., Conway, S. J., Smyth, S. S. \& Granger, D. N. (2010) Periostin mediates vascular smooth muscle cell migration through the integrins alphavbeta3 and alphavbeta5 and focal adhesion kinase (FAK) pathway. Atherosclerosis 208, 358-365.

Ling, L., Cheng, Y., Ding, L. \& Yang, X. (2014) Association of serum periostin with cardiac function and short-term prognosis in acute myocardial infarction patients. PLoS One 9, e88755.

Litvin, J., Selim, A. H., Montgomery, M. O., Lehmann, K., Rico, M. C., Devlin, H., Bednarik, D. P. \& Safadi, F. F. (2004) Expression and function of periostin-isoforms in bone. Journal of Cellular Biochemistry 92, 1044-1061.

Maruhashi, T., Kii, I., Saito, M. \& Kudo, A (2010) Interaction between periostin and BMP1 promotes proteolytic activation of lysyl oxidase. Journal of Biological Chemistry $\mathbf{2 8 5}$, 13294-13303.

Matsumoto, H. (2014) Serum periostin: a novel biomarker for asthma management. Allergology International 63, 153-160

Nakajima, M., Honda, T., Miyauchi, S. \& Yamazaki, K. (2014) Th2 cytokines efficiently stimulate periostin production in gingival fibroblasts but periostin does not induce an inflammatory response in gingival epithelial cells. Archives of Oral Biology 59, 93-101.

Nishiyama, T., Kii, I., Kashima, T. G., Kikuchi, Y., Ohazama, A., Shimazaki, M. Fukayama, M. \& Kudo, A. (2011) Delayed re-epithelialisation in periostin-deficient mice during cutaneous wound healing. PLoS One 6, e18410.

Norris, R. A., Damon, B., Mironov, V., Kasyanov, V., Ramamurthi, A., Moreno-Rodriguez, R., Trusk, T., Potts, J. D., Goodwin, R. L., Davis, J., Hoffman, S., Wen, X., Sugi, Y., Kern, C. B., Mjaatvedt, C. H., Turner, D. K. Oka, T., Conway, S. J., Molkentin, J. D., Forgacs, G. \& Markwald, R. R. (2007) Periostin regulates collagen fibrillogenesis and the biomechanical properties of connective tissues. Journal of Cellular Biochemistry 101, 695-711.

Nuzzo, P. V., Buzzatti, G., Ricci, F., Rubagotti, A., Argellati, F., Zinoli, L. \& Boccardo, F. (2014) Periostin: A novel prognostic and therapeutic target For genitourinary cancer? Clinical Genitourinary Cancer. 12, 301-311. 
Okamoto, M., Hoshino, T., Kitasato, Y., Sakazaki, Y., Kawayama, T., Fujimoto, K., Ohshima, K., Shiraishi, H., Uchida, M., Ono, J., Ohta, S., Kato, S., Izuhara, K. \& Aizawa, H. (2011) Periostin, a matrix protein, is a novel biomarker for idiopathic interstitial pneumonias. European Respiratory Journal 37, 1119-1127.

Padial-Molina, M., Marchesan, J. T., Taut, A. D., Jin, Q., Giannobile, W. V. \& Rios, H. F. (2012a) Methods to validate tooth-supporting regenerative therapies. Methods in Molecular Biology 887, 135-148.

Padial-Molina, M., Volk, S. L. \& Rios, H. F. (2014) Periostin increases migration and proliferation of human periodontal ligament fibroblasts challenged by tumor necrosis factor -alpha and Porphyromonas gingivalis lipopolysaccharides. Journal of Periodontal Research 49, 405-414.

Padial-Molina, M., Volk, S. L., Rodriguez, J. C. Marchesan, J. T., Galindo-Moreno, P. \& Rios, H. F. (2013) Tumor necrosis factor-alpha and Porphyromonas gingivalis lipopolysaccharides decrease periostin in human periodontal ligament fibroblasts. Journal of Periodontology $\mathbf{8 4}$ 694-703.

Padial-Molina, M., Volk, S. L., Taut, A. D., Giannobile, W. V. \& Rios, H. F. (2012b) Periostin is down-regulated during periodontal inflammation. Journal of Dental Research 91, 1078-1084.

Park, C. H., Rios, H. F., Jin, Q., Sugai, J. V., Padial-Molina, M., Taut, A. D., Flanagan, C. L., Hollister, S. J. \& Giannobile, W. V. (2012) Tissue engineering bone-ligament complexes using fiber-guiding scaffolds. Biomaterials 33, 137145.

Park, C. H., Rios, H. F., Taut, A. D., PadialMolina, M., Flanagan, C. L., Pilipchuk, S. P., Hollister, S. J. \& Giannobile, W. V. (2014) Image-based, fiber guiding scaffolds: a platform for regenerating tissue interfaces. Tissue Engineering Part $C$ Methods 20, 533-542.

Polizzotti, B. D., Arab, S. \& Kuhn, B. (2012) Intrapericardial delivery of gelfoam enables the targeted delivery of Periostin peptide after myocardial infarction by inducing fibrin clot formation. PLoS One 7, e36788.

Rios, H. F., Ma, D., Xie, Y., Giannobile, W. V., Bonewald, L. F., Conway, S. J. \& Feng, J. Q. (2008) Periostin is essential for the integrity and function of the periodontal ligament during occlusal loading in mice. Journal of Periodontology 79, 1480-1490.

Rosselli-Murai, L. K., Almeida, L. O., Zagni, C., Galindo-Moreno, P., Padial-Molina, M., Volk, S. L., Murai, M. J., Rios, H. F., Squarize, C. H. \& Castilho, R. M. (2013) Periostin responds to

\section{Clinical Relevance}

Scientific rationale for the study: Periodontal disease is a multifactorial process. Extracellular matrix and matricellular interactions are key for tissue homeostasis and, therefore, highly relevant to disease pathogenesis and tissue healing. Periostin, a highly specific matricel- mechanical stress and tension by activating the MTOR signalling pathway. PLoS One 8, e83580.

Rousseau, J. C., Sornay-Rendu, E., Bertholon, C., Chapurlat, R. \& Garnero, P. (2014) Serum periostin is associated with fracture risk in postmenopausal women: a 7-year prospective analysis of the OFELY study. Journal of Clinical Endocrinology and Metabolism 99, 2533 2539.

Saito, Y., Yoshizawa, T., Takizawa, F., Ikegame, M., Ishibashi, O., Okuda, K., Hara, K., Ishibashi, K., Obinata, M. \& Kawashima, H. (2002) A cell line with characteristics of the periodontal ligament fibroblasts is negatively regulated for mineralisation and Runx $2 / \mathrm{Cbfa} 1 / \mathrm{Osf} 2$ activity, part of which can be overcome by bone morphogenetic protein-2. Journal of Cell Science 115, 4191-4200

Sasaki, H., Yu, C. Y., Dai, M., Tam, C., Loda, M., Auclair, D., Chen, L. B. \& Elias, A. (2003) Elevated serum periostin levels in patients with bone metastases from breast but not lung cancer. Breast Cancer Research and Treatment 77, 245-252.

Snider, P., Hinton, R. B., Moreno-Rodriguez, R. A., Wang, J., Rogers, R., Lindsley, A., Li, F., Ingram, D. A., Menick, D., Field, L., Firulli, A. B., Molkentin, J. D., Markwald, R. \& Conway, S. J. (2008) Periostin is required for maturation and extracellular matrix stabilisation of noncardiomyocyte lineages of the heart. Circulation Research 102, 752-760.

Suzuki, H., Amizuka, N., Kii, I., Kawano, Y., Nozawa-Inoue, K., Suzuki, A., Yoshie, H., Kudo, A. \& Maeda, T. (2004) Immunohistochemical localisation of periostin in tooth and its surrounding tissues in mouse mandibles during development. The Anatomical Record. Part A, Discoveries in Molecular, Cellular, and Evolutionary Biology 281, 1264-1275.

Takeshita, S., Kikuno, R., Tezuka, K. \& Amann, E. (1993) Osteoblast-specific factor 2: cloning of a putative bone adhesion protein with homology with the insect protein fasciclin I. The Biochemical Journal 294 (Pt 1), 271-278.

Tian, B., Zhang, Y. \& Zhang, J. (2014) Periostin is a new potential prognostic biomarker for glioma. Tumour Biology. 35, 5877-5883.

Utispan, K., Sonongbua, J., Thuwajit, P., ChauIn, S., Pairojkul, C., Wongkham, S. \& Thuwajit, C. (2012) Periostin activates integrin alpha5betal through a PI3K/AKTdependent pathway in invasion of cholangiocarcinoma. In ternational Journal of Oncology 41, 1110-1118.

Wang, Z. \& Ouyang, G. (2012) Periostin: A bridge between cancer stem cells and their metastatic niche. Cell Stem Cell 10, 111-112.
Yamada, S., Tauchi, T., Toshihito, A., Maeda, K., Kajikawa, T., Yanagita, M. \& Murakami, S. (2014) Characterisation of a novel periodontal ligament-specific periostin isoform. Journal of Dental Research 93, 891-897.

Zhu, M., Fejzo, M. S., Anderson, L., Dering, J., Ginther, C., Ramos, L., Gasson, J. C., Karlan, B. Y. \& Slamon, D. J. (2010) Periostin promotes ovarian cancer angiogenesis and metastasis. Gynecologic Oncology 119, 337-344.

\section{Supporting Information}

Additional Supporting Information may be found in the online version of this article:

Appendix S1. Supplementary methods.

Figure S1. Percentages of patients classified by different categories of categorical clinical variables: (a) Oral hygiene; (b) Soft tissue index; (c) Discomfort; and (d) Wound healing index.

Figure S2. Levels of periostin protein in tissue in $\mathrm{ng} / \mathrm{ml}$ (mean $\pm \mathrm{SEM}$ ) for healthy (blue) and disease (red) patients.

Figure S3. Levels of periostin, $\beta \mathrm{IG}$ H3, collagen I, twist and TGF- $\beta 1$ mRNA (mean \pm SEM) in tissues for healthy and disease patients.

Figure S4. Levels of CRP over time in serum in $\mathrm{mg} / \mathrm{dl}$ (mean $\pm \mathrm{SEM}$ ) for healthy (blue) and disease (red) patients.

Address:

Hector F. Rios

1011 North University Ave

Office 3349

Ann Arbor

MI 48109-1078

USA

E-mail: hrios@umich.edu

lular protein of the periodontal ligament, has been shown to be determinant in previous animal studies.

Principal findings: This study indicates that periostin plays a determinant role in periodontal tissue healing as it increases after periodontal surgery in gingival crevicular fluid.
Practical implications: Periostin basal levels could potentially be used as a biomarker of periodontal disease susceptibility and a healing potential determinant. Future studies will confirm its promising application as a regenerative agent. 